\title{
Modeling the Cost Savings of Continuous Pulse Oximetry and Capnography Monitoring of United States General Care Floor Patients Receiving Opioids Based on the PRODIGY Trial
}

\author{
Ashish K. Khanna • Carla R. Jungquist • Wolfgang Buhre · \\ Roy Soto · Fabio Di Piazza - Leif Saager on behalf of the PRediction of Opioid-induced respiratory Depression In \\ patients monitored by capnoGraphY (PRODIGY) Group Investigators
}

Received: April 7, 2021 / Accepted: May 6, 2021 / Published online: May 24, 2021

(C) The Author(s) 2021

\section{ABSTRACT}

Introduction: Despite the high incidence of respiratory depression on the general care floor and evidence that continuous monitoring improves patient outcomes, the cost-benefit of continuous pulse oximetry and capnography

The members of the PRediction of Opioid-induced respiratory Depression In patients monitored by capnoGraphY (PRODIGY) Group Investigators are listed in acknowledgements.

Supplementary Information The online version contains supplementary material available at https:// doi.org/10.1007/s12325-021-01779-7.

A. K. Khanna $(\bowtie)$

Department of Anesthesiology, Section on Critical Care Medicine, Wake Forest School of Medicine, Wake Forest Center for Biomedical Informatics, Critical Illness, Injury and Recovery Research Center (CIIRRC), Medical Center Boulevard, WinstonSalem, NC 27157, USA

e-mail: akhanna@wakehealth.edu

\section{A. K. Khanna}

Outcomes Research Consortium, Cleveland, $\mathrm{OH}$, USA

C. R. Jungquist

University at Buffalo School of Nursing, Buffalo, NY, USA

W. Buhre

Department of Anesthesiology, University Medical

Center, Maastricht, The Netherlands monitoring of general care floor patients remains unknown. This study modeled the cost and length of stay savings, investment breakeven point, and likelihood of cost savings for continuous pulse oximetry and capnography monitoring of general care floor patients at risk for respiratory depression.

Methods: A decision tree model was created to compare intermittent pulse oximetry versus continuous pulse oximetry and capnography monitoring. The model utilized costs and

\author{
R. Soto \\ Department of Anesthesiology, Beaumont Hospital, \\ Royal Oak, MI, USA \\ F. Di Piazza \\ Medtronic Core Clinical Solutions, Study and \\ Scientific Solutions, Rome, Italy \\ L. Saager \\ Department of Anesthesiology, University of \\ Michigan Medical School, Ann Arbor, MI, USA \\ L. Saager \\ Department of Anesthesiology, University Medical \\ Center Goettingen, Goettingen, Germany
}


outcomes from the PRediction of Opioid-induced respiratory Depression In patients monitored by capnoGraphY (PRODIGY) trial, and was applied to a modeled cohort of 2447 patients receiving opioids per median-sized United States general care floor annually.

Results: Continuous pulse oximetry and capnography monitoring of high-risk patients is projected to reduce annual hospital cost by $\$ 535,531$ and cumulative patient length of stay by 103 days. A $1.5 \%$ reduction in respiratory depression would achieve a break-even investment point and justify the investment cost. The probability of cost saving is $\geq 80 \%$ if respiratory depression is decreased by $\geq 17 \%$. Expansion of continuous monitoring to high- and intermediate-risk patients, or to all patients, is projected to reach a break-even point when respiratory depression is reduced by $2.5 \%$ and $3.5 \%$, respectively, with $\mathrm{a} \geq 80 \%$ probability of cost savings when respiratory depression decreases by $\geq 27 \%$ and $\geq 31 \%$, respectively.

Conclusion: Compared to intermittent pulse oximetry, continuous pulse oximetry and capnography monitoring of general care floor patients receiving opioids has a high chance of being cost-effective.

Trial Registration: www.clinicaltrials.gov, Registration ID: NCT02811302.

Keywords: Break-even analysis; Capnography;

Continuous monitoring; Cost savings; Economic model; General care floor; Healthcare economics; Pulse oximetry; Respiratory compromise; Respiratory depression

\section{Key Summary Points}

Why carry out this study?

Respiratory depression occurs in $46 \%$ of patients receiving opioids on the general care floor, where standard of care monitoring consists of intermittent pulse oximetry spot-checks.

Continuous pulse oximetry and capnography monitoring can detect respiratory depression, but the cost-benefit of continuous pulse oximetry and capnography monitoring is unknown.

The purpose of this study was to model the cost and length of stay savings, investment break-even point, and likelihood of cost savings for continuous pulse oximetry and capnography monitoring of general care floor patients at risk for respiratory depression.

\section{What was learned from the study?}

Continuous pulse oximetry and capnography monitoring of high-risk patients could reduce annual hospital cost by $\$ 535,531$ and cumulative patient length of stay by 103 days, reaching a break-even investment point when the incidence of respiratory depression decreases by $1.5 \%$. 
Compared to intermittent pulse oximetry, continuous pulse oximetry and capnography monitoring of general care floor patients receiving opioids has a high chance of being cost-effective.

\section{PLAIN LANGUAGE SUMMARY}

Respiratory depression occurs when a person has an abnormally slow breath rate, low oxygen saturation, low or high concentration of exhaled carbon dioxide, or stops breathing intermittently. This condition occurs in $46 \%$ of patients receiving opioids in medical and surgical hospital units. Respiratory depression can be detected using continuous respiratory monitoring to measure respiratory rate, heart rate, blood oxygen saturation, and exhaled carbon dioxide. However, the cost-benefit of continuous respiratory monitoring in patients hospitalized in medical and surgical units is unknown. We created an economic model to predict differences in hospital cost and patient length of stay when using continuous respiratory monitoring, compared to intermittent blood oxygen saturation and heart rate monitoring. This model predicts the break-even point where the cost of investing in monitoring technology will equal the costs saved by preventing respiratory depression, and evaluates the chance that continuous respiratory monitoring will be cost saving. If patients at highest risk for respiratory depression are continuously monitored, the model projects annual hospital cost will decrease by $\$ 535,531$, and total length of stay of all patients will decrease by 103 days. The cost of investing in monitoring is predicted to equal the cost savings if respiratory depression decreases by $1.5 \%$, and there is a high probability of the investment being cost saving. The model also predicts that continuous monitoring will reduce annual cost and length of stay if patients at high and intermediate risk, or if all patients, undergo continuous respiratory monitoring. Overall, continuous respiratory monitoring has a high chance of being costeffective.

\section{DIGITAL FEATURES}

This article is published with digital features, including a summary slide and plain language summary, to facilitate understanding of the article. To view digital features for this article go to https://doi.org/10.6084/m9.figshare. 14541750 .

\section{INTRODUCTION}

Respiratory depression is common on hospital general care floors, where nurses traditionally assess vital signs every 4-6 h [1-3]. However, compared to intermittent assessment of respiratory status, continuous electronic monitoring detects significantly more cardiorespiratory events $[1,4]$. For example, $20 \%$ of all postoperative patients experience significant time under hypoxemic thresholds, and almost all go undetected with intermittent monitoring [1]. Low respiratory rate occurs in $41 \%$ of patients in the post-anesthesia care unit and general care floor [5].

Recently, the PRediction of Opioid-induced respiratory Depression In patients monitored by capnoGraphY (PRODIGY) trial reported that $46 \%$ of postsurgical and medical patients receiving parenteral opioids experienced respiratory depression episodes [2]. This prospective trial comprehensively assessed respiratory depression, monitoring patients with blinded continuous pulse oximetry and capnography, including strict time and threshold cutoffs to define respiratory depression [2]. The PRODIGY trial found that adverse events requiring rescue action or prolonged hospitalization occurred more often in patients with monitor-detected respiratory depression episodes $[2,6]$. This is consistent with prior reports using continuous pulse oximetry or capnography only $[1,5]$, further substantiating that respiratory depression may be preventable with better monitoring and earlier intervention $[2,6,7]$.

Respiratory opioid-related adverse events (ORADE) are costly to healthcare systems [8-10]. In particular, unrecognized respiratory depression contributes to poor patient outcomes and longer lengths of stay that increase 
healthcare cost $[6,11-13]$. Compared to United States (US) general care floor patients without respiratory depression, hospital cost is $\$ 3686$ higher in patients with respiratory depression [6]. Although professional organizations and their clinical practice guidelines recommend the use of electronic respiratory monitoring, the majority of US hospitals do not have an adequate supply of devices $[14,15]$. This is because despite current evidence that early recognition of respiratory depression can potentially decrease hospital cost, instituting continuous monitoring is perceived as requiring significant financial investment [16].

The purpose of this analysis was to evaluate the economic value of continuous monitoring by modeling the investment break-even point and likelihood of cost savings with continuous pulse oximetry and capnography monitoring compared to intermittent pulse oximetry monitoring of general care floor patients receiving opioids.

\section{METHODS}

A model was developed to estimate the investment break-even point and likelihood of cost savings associated with implementation of continuous pulse oximetry and capnography monitoring of US general care floor patients at risk for respiratory depression. The model, which follows good practice guidelines (International Society of Pharmacoeconomics and Outcomes Research) [17], was created using a decision tree framework in Excel (Microsoft, Redmond, WA). The model considers a budget holder perspective for a median-sized US hospital and projects cost over a 1-year time horizon.

\section{Model Structure}

The model was created to compare costs and outcomes for (1) standard of care intermittent pulse oximetry monitoring versus (2) continuous respiratory monitoring with pulse oximetry and capnography of medical and surgical patients receiving opioids on the general care floor. Within the continuously monitored group, the model simulates outcomes for three scenarios: monitoring of patients at (1) high risk, (2) high and intermediate risk, or (3) any level (high, intermediate, or low) risk of respiratory depression, determined using the PRODIGY score (Fig. 1) [2]. This model considers three PRODIGY trial outcomes: (1) incidence of respiratory depression episodes, (2) length of stay, and (3) total hospital admission costs. Owing to lack of interventional data, the effect of implementing continuous monitoring was modeled from $0 \%$ to $100 \%$ reduction in respiratory depression.

\section{PRODIGY Trial}

The PRODIGY trial enrolled 1495 patients age $\geq 18,20$, and 21 across the USA and Europe, Japan, and Singapore, respectively. This post hoc analysis included only US patients who received parenteral opioids and underwent blinded continuous pulse oximetry and capnography monitoring on the general care floor $(N=758)[2,18]$. Continuous monitoring continued up to $48 \mathrm{~h}$ using the Capnostream ${ }^{\mathrm{TM}}$ 20 p or 35 portable bedside monitor (Medtronic, Boulder, CO). The PRODIGY trial was conducted following institutional review board or ethics committee approval, with written informed consent collected from all patients. Respiratory depression episodes included respiratory rate $\leq 5$ breaths/minute, oxygen saturation $\leq 85 \%$, or end-tidal carbon dioxide $\leq 15$ or $\geq 60 \mathrm{mmHg}$ for $\geq 3 \mathrm{~min}$; apnea episode for $>30 \mathrm{~s}$; or any respiratory opioid-related adverse event $[2,18]$. Each patient was retrospectively assessed for the risk of respiratory depression using the PRODIGY score, which was derived from the PRODIGY dataset using a multivariable logistic regression model [18]. The model includes five independent patient characteristics, including age $\geq 60$ in decades, male sex, sleep disordered breathing, opioid naivety, and chronic heart failure [2]. Each variable is assigned a point value, and patients can be easily evaluated for their risk of respiratory depression based on the sum of the points assigned, with low ( $<8$ points), intermediate ( $\geq 8$ and $<15$ points), and high ( $\geq 15$ points) 
risk categories $[2,6]$. Since the PRODIGY dataset was used to derive the PRODIGY score, for the purposes of this analysis, each PRODIGY patient was retrospectively assigned a PRODIGY score based on his or her age, sex, opioid naivety, and presence of sleep disordered breathing and chronic heart failure [6].

Patients missing length of stay and hospital cost data $(N=11)$ or data required to determine PRODIGY score $(N=9)$ were excluded [6]. To determine the influence of patient outliers on the model, the analysis was performed using all patients with hospital costs available, and separately using a population excluding patient outliers for hospital cost or length of stay, identified using Cook's distance [6].

\section{Modeled Cohort}

The model extrapolates PRODIGY outcomes to a cohort of general care floor patients receiving opioids in a median-sized US hospital. On the basis of the 2018 Premier ${ }^{\circledR}$ Healthcare Database, $90 \%$ of surgical patients and $45 \%$ of medical patients on US general care floors receive opioids, with a median 2447 opioid-receiving patients per hospital per year $(95 \%$ CI 2092-2802) (Table S1 in Supplementary Material). This is consistent with recent literature [10, 19-23].

\section{Model Inputs and Data Sources}

Twenty percent $(N=171 / 835), 34 \%(N=300 /$ $874)$, and $60 \%(N=442 / 738)$ of the modeled cohort was allocated to low-, intermediate-, and high-risk respiratory depression groups, matching the PRODIGY trial distribution (Tables 1 and 2). PRODIGY cost and length of stay data were model inputs (Table 1) [6]. Discount rates, to determine net present value of costs and outcomes, and inflationary adjustments were not applied because of the model's short time horizon and the recency of the PRODIGY trial. The same model structure was applied separately utilizing inputs from the PRODIGY cohort excluding outliers (Table S2 in Supplementary Material).

For intermittent pulse oximetry monitoring, device pricing consisted of a multiparameter monitor (\$3000) prorated over 7 years and a reusable pulse oximetry sensor (\$65). A US hospital with 27 opioid-receiving general care floor patients (Premier ${ }^{\circledR}$ Healthcare Database) would need three multiparameter monitors and two reusable pulse oximetry sensors per monitor, resulting in a device cost of $\$ 0.68$ per patient stay. Continuous pulse oximetry and capnography device pricing consisted of a Capnostream $^{\mathrm{TM}}$ portable respiratory monitor (\$4300, Medtronic, Boulder, CO) prorated over 7 years, a Microstream ${ }^{\mathrm{TM}}$ capnography filterline

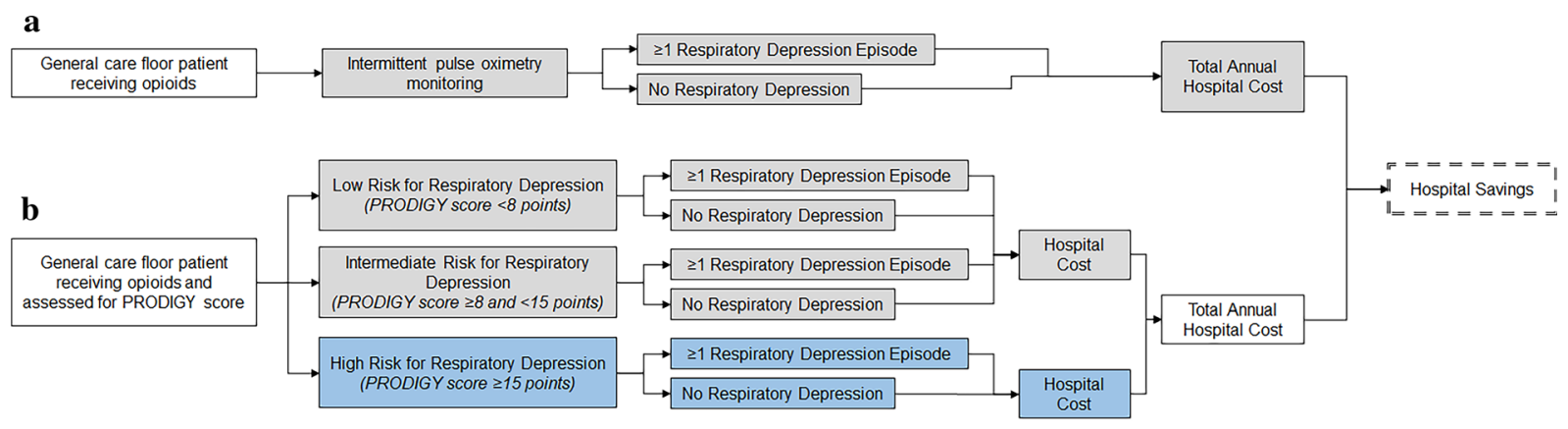

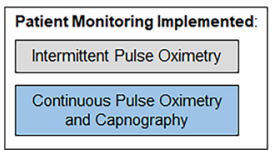

Fig. 1 Model framework, distinguishing between total annual hospital cost for a standard of care intermittent pulse oximetry monitoring and $\mathbf{b}$ implementation of continuous pulse oximetry and capnography monitoring based on patient PRODIGY score 
(\$14.50, Medtronic, Boulder, CO), and a disposable Nellcor ${ }^{\mathrm{TM}}$ pulse oximetry sensor $(\$ 8.50$, Medtronic, Boulder, CO), for a device cost of $\$ 52.73$ per monitored patient.

In the base case, the percentage reduction in patients with $\geq 1$ respiratory depression episode was conservatively assumed at $20 \%$ reduction based on available literature, which reports a $34 \%$ risk reduction for intensive care unit (ICU) transfer upon implementation of continuous pulse oximetry monitoring, and reduction of severe opioid-related adverse events from 3.1/ 10,000 patients to $0.6 / 10,000$ patients and a $50 \%$ decrease in rapid response calls due to opioid-induced respiratory depression following implementation of continuous capnography monitoring [16, 24, 25]. Break-even and sensitivity analyses did not rely on this assumption.

\section{Modeled Scenarios}

In the base case, the model evaluates annual hospital cost and cumulative patient length of stay using intermittent pulse oximetry monitoring of all general care floor patients receiving opioids, versus continuous pulse oximetry and capnography monitoring of general care floor patients with high respiratory depression risk receiving opioids (Fig. 1). Additional scenarios evaluate the same outcomes, comparing intermittent pulse oximetry monitoring versus continuous respiratory monitoring of general care floor patients receiving opioids with high and intermediate respiratory depression risk, or any level respiratory depression risk. Break-even analysis simulated the minimum requirements needed to justify the investment, modeling cost and length of stay savings as a function of the percentage respiratory depression reduction from $0 \%$ to $100 \%$.

\section{Sensitivity Analysis}

Probabilistic sensitivity analysis was performed using 1000 Monte Carlo simulations [26] to determine the probability of continuous pulse oximetry and capnography monitoring being cost saving. The model estimates the investment break-even point and likelihood of cost savings when respiratory depression cases decrease from $0 \%$ to $100 \%$. Parameters were assigned a probability distribution, including distinction of costs (gamma distribution), and epidemiological parameters (beta distributions), and accounted for the standard error of PRODIGY dataset parameters. One-way deterministic sensitivity analysis was performed to examine the effects of model parameters on cost and length of stay outcomes. The uncertainty of each parameter was determined using variability estimated from the PRODIGY dataset.

\section{RESULTS}

\section{Base Case}

Across all general care floor patients receiving opioids, continuous pulse oximetry and capnography monitoring of patients with high risk for respiratory depression would result in $\$ 535,531$ annual savings and a cumulative length of stay decrease of 103 days per year (Table 2), compared to standard of care intermittent pulse oximetry monitoring. This assumes a $20 \%$ respiratory depression reduction and an annual general care floor volume of 2447 patients receiving opioids per mediansized hospital (Table S1 in Supplementary Material). In this scenario, the model predicts that a $1.5 \%$ reduction in respiratory depression would achieve an investment break-even point, with cost and length of stay savings increasing linearly as respiratory depression decreases (Fig. 2a-b). Reducing respiratory depression by $10 \%, 20 \%$, and $30 \%$ would decrease hospital costs by $\$ 341, \$ 726$, and $\$ 1110$ per patient, respectively (Fig. S1A in Supplementary Material).

\section{Additional Monitoring Scenarios}

A second scenario modeled the cost savings of continuously monitoring patients at high and intermediate respiratory depression risk with pulse oximetry and capnography, compared to intermittent pulse oximetry monitoring of all patients. In this scenario, cumulative patient 
Table 1 Model inputs

\begin{tabular}{|c|c|c|c|}
\hline PRODIGY risk score & Low $(<8$ points $)$ & $\begin{array}{l}\text { Intermediate }(\geq 8 \text { and } \\
<15 \text { points })\end{array}$ & $\begin{array}{l}\text { High }(\geq 15 \\
\text { points) }\end{array}$ \\
\hline Patients in risk group $(N=758)$ & $34 \%(258 / 758)$ & $36 \%(273 / 758)$ & $30 \%(227 / 758)$ \\
\hline $\begin{array}{l}\text { Patients with respiratory depression in risk group } \\
\qquad(N=758)\end{array}$ & $20 \%(52 / 258)$ & $34 \%(93 / 273)$ & $60 \%(136 / 227)$ \\
\hline \multicolumn{4}{|l|}{ Mean length of stay (days) $(N=758)$} \\
\hline Patients without respiratory depression episodes & $5.2 \pm 6.4$ & $6.0 \pm 6.1$ & $6.4 \pm 7.8$ \\
\hline Patients with $\geq 1$ respiratory depression episode & $6.8 \pm 9.4$ & $6.8 \pm 10.7$ & $7.5 \pm 9.1$ \\
\hline \multicolumn{4}{|l|}{ Mean hospital cost $(N=411)$} \\
\hline Patients without respiratory depression episodes & $\$ 18,633 \pm 14,050$ & $\$ 20,331 \pm 14,594$ & $\$ 18,608 \pm 9714$ \\
\hline Patients with $\geq 1$ respiratory depression episode & $\$ 22,316 \pm 13,679$ & $\$ 22,272 \pm 14,661$ & $\$ 25,057 \pm 19,490$ \\
\hline $\begin{array}{l}\text { Median number of patients receiving opioids on general } \\
\text { care floor per hospital per year }\end{array}$ & 2447 & & \\
\hline \multicolumn{4}{|l|}{ Monitoring cost per patient } \\
\hline Intermittent pulse oximetry & $\$ 0.68$ per stay & & \\
\hline Continuous pulse oximetry and capnography & $\$ 52.73$ per stay & & \\
\hline
\end{tabular}

Risk score distributions, length of stay, and healthcare cost are from US patients enrolled in the PRODIGY trial. Median number of patients per hospital per year is sourced from the Premier ${ }^{\circledR}$ Healthcare Database, and Medtronic provided monitoring cost estimates

length of stay would decrease by 152 days, with an annual savings of $\$ 606,463$ (Table 2). The reduction in respiratory depression required to reach a break-even point was $2.5 \%$ (Fig. $2 \mathrm{a}$ ).

The third scenario, in which all patients receiving opioids, regardless of risk for respiratory depression, undergo continuous respiratory monitoring, projected a cumulative length of stay savings of 204 days, with $\$ 688,221$ in annual savings, compared to intermittent pulse oximetry monitoring of the same patients (Table 2). The projected break-even point would occur when respiratory depression decreased by $3.5 \%$ (Fig. 2a). These continuous monitoring scenarios are expected to linearly decrease perpatient cost and length of stay as respiratory depression decreases (Fig. S1A in Supplementary Material, Fig. 2b).

\section{Monitoring Scenarios Excluding Patient Outliers}

To evaluate the impact of patient outliers, a separate analysis was performed using the PRODIGY cohort excluding patient outliers. Compared to intermittent pulse oximetry monitoring on all patients, continuous pulse oximetry and capnography monitoring of patients with high respiratory depression risk would result in a 119-day reduction in cumulative length of stay and an annual hospital cost savings of \$257,561 (Table S3 in Supplementary Material). A 3\% reduction in respiratory depression would achieve an investment breakeven point (Fig. S2A in Supplementary Material), with a $\$ 355$ per-patient cost savings if respiratory depression decreased 20\% (Fig. S1B in Supplementary Material). Similarly, the model excluding outliers predicts length of stay reductions of 183 and 216 days and annual 


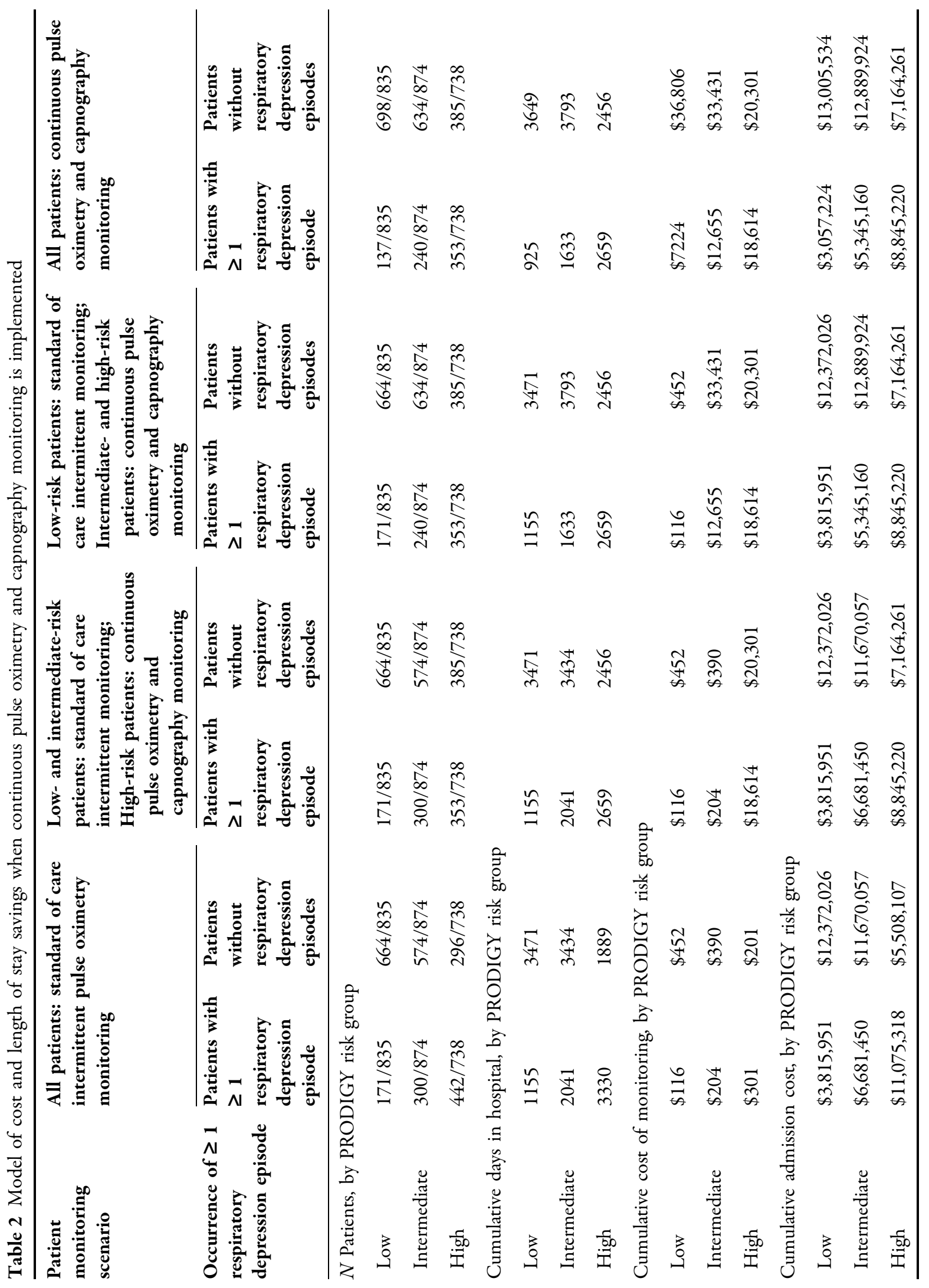




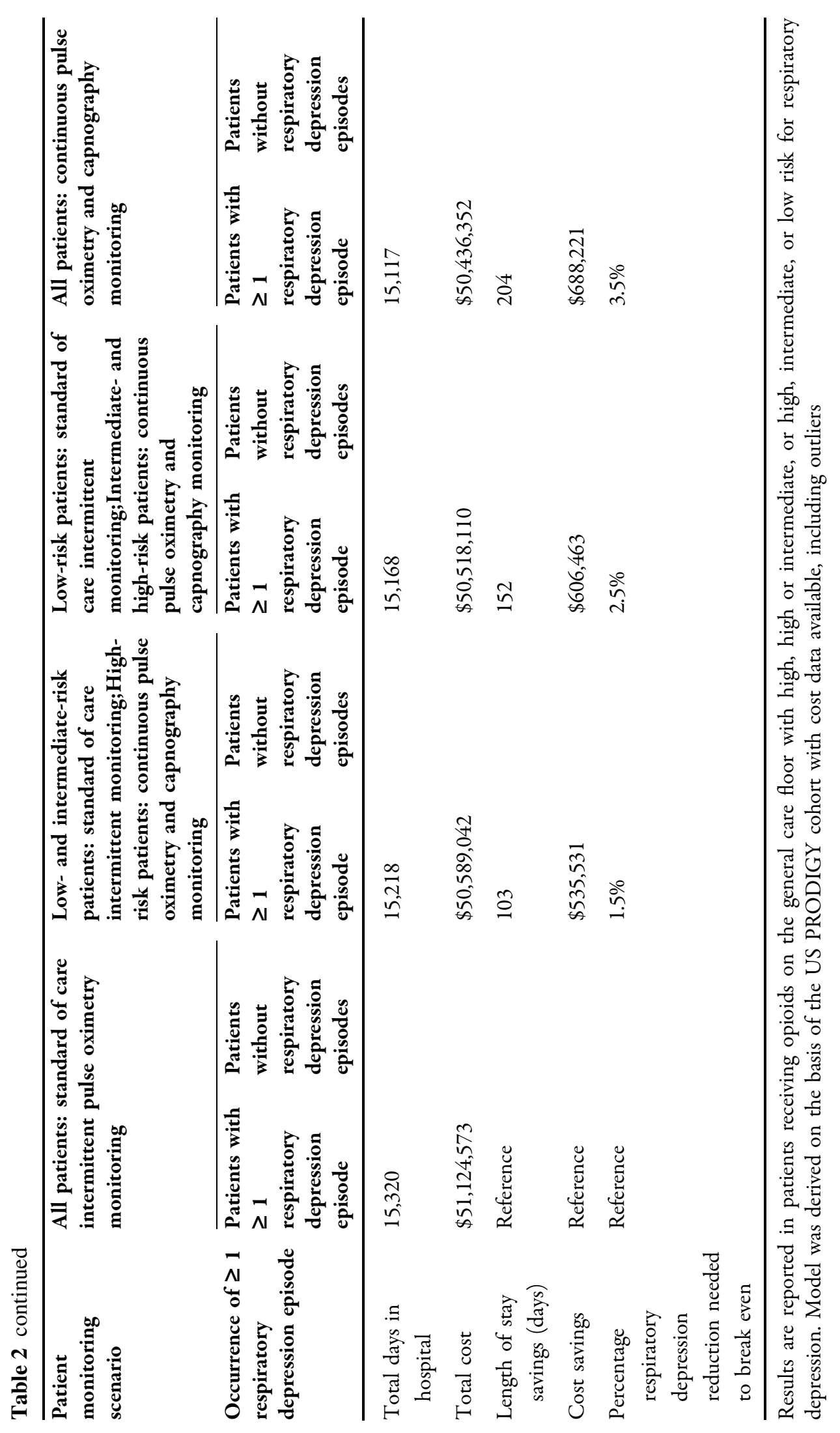




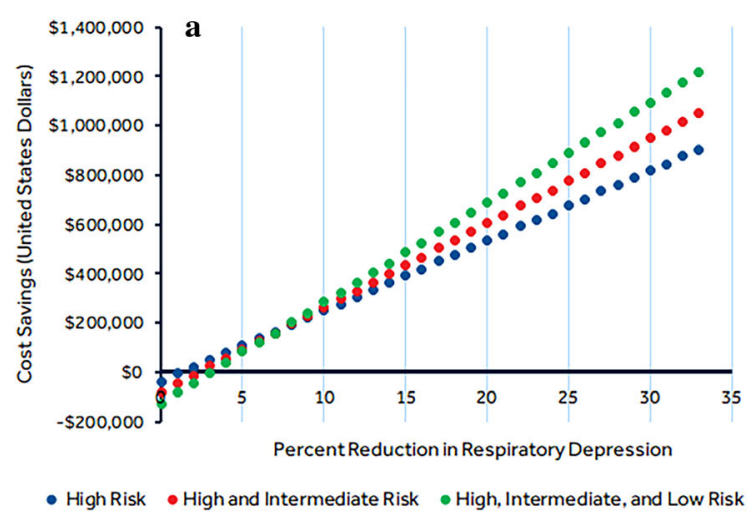

Fig. 2 a Annual cost savings (US dollars) and b length of stay reduction predicted following implementation of continuous pulse oximetry and capnography monitoring on patients with high (blue line), high or intermediate (red

savings of $\$ 380,405$ and $\$ 497,734$ following implementation of continuous respiratory monitoring on patients with high and intermediate risk of respiratory depression, or on all patients, respectively (Table S3 in Supplementary Material). In these scenarios, the breakeven point would occur when respiratory depression decreases by $4-4.5 \%$, with a linear decrease in length of stay as respiratory depression decreases (Fig. S2A-B in Supplementary Material).

\section{Probabilistic Sensitivity Analysis}

To achieve $a \geq 80 \%$ probability of cost savings when patients with high respiratory depression risk undergo continuous respiratory monitoring, a decrease in respiratory depression $\geq 17 \%$ would be needed. This increases to $\mathrm{a} \geq 96 \%$ probability of cost savings if respiratory depression is decreased by $\geq 30 \%$ (Fig. 3). In scenarios in which continuous monitoring is applied to high- and intermediate-risk patients, or to all patients, $\mathrm{a} \geq 27 \%$ and $\geq 31 \%$ reduction in respiratory depression would be needed to achieve $a \geq 80 \%$ probability of being cost saving, respectively (Fig. 3).

Probabilistic sensitivity analysis of the model using the PRODIGY cohort excluding outliers projects that when patients with high, high or intermediate, or any respiratory depression risk

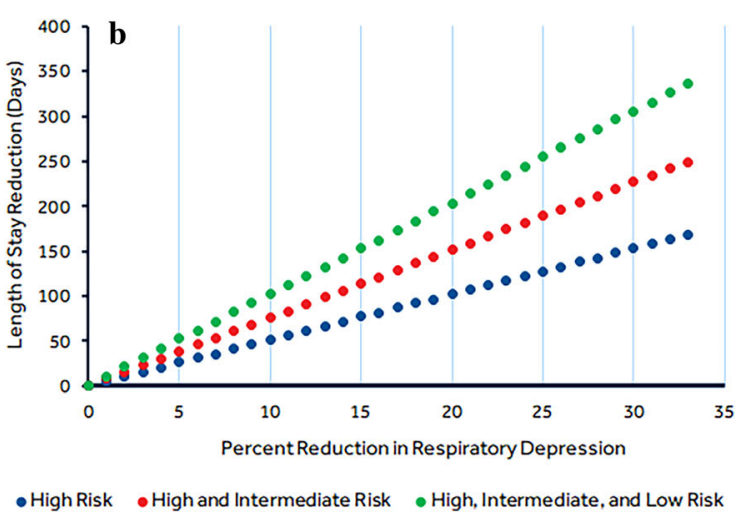

line), or high, intermediate, or low (green line) risk for respiratory depression. Model was derived the on basis of the US PRODIGY cohort with cost data available, including outliers

undergo continuous monitoring, a respiratory depression decrease of $\geq 27 \%, \geq 35 \%$, and $\geq 35 \%$, respectively, would be needed to achieve a $\geq 80 \%$ probability of being cost saving (Fig. S3 in Supplementary Material).

\section{Deterministic Sensitivity Analysis}

Model drivers were evaluated using one-way deterministic sensitivity analysis. For the model based on the PRODIGY cohort with outliers, admission cost of high- and intermediate-risk patients with respiratory depression had the strongest influence on model outcomes (Fig. S4A in Supplementary Material). Other drivers included the admission cost of high- and intermediate-risk patients without respiratory depression, the cost of low-risk patients with respiratory depression, and the number of general care floor patients receiving opioids per year. For the model excluding patient outliers, the main model drivers were similar (Fig. S4B in Supplementary Material). Monitoring device costs had a minimal influence on results.

\section{DISCUSSION}

Our analysis suggests that a reduction of $\geq 1.5 \%, \geq 2.5 \%$, and $\geq 3.5 \%$ in respiratory depression episodes would justify the 
investment for continuous pulse oximetry and capnography monitoring when continuously monitoring high, high and intermediate, or all patients, respectively. Projected annual cost savings, assuming a $20 \%$ reduction in respiratory depression, would be $\$ 535,531$ to $\$ 688,221$, and reduction in cumulative length of stay would be 103-204 days annually, depending on the PRODIGY risk group(s) monitored. Furthermore, implementation of continuous respiratory monitoring has $\geq 80 \%$ probability of being cost saving if respiratory depression is reduced by $\geq 17 \%, \geq 27 \%$, and $\geq 31 \%$ if implemented on high-risk, high- and intermediate-risk, and all patients, respectively. Importantly, implementation of continuous monitoring alone will not decrease respiratory depression or adverse events, but can alert bedside providers to respiratory depression and facilitate early intervention. A true decrease in respiratory depression is dependent on how bedside providers respond to these alerts.

Our analysis showed that the top model drivers include the cost burden of respiratory depression and general care floor volume, not the cost of monitoring equipment. This may be explained by the high cost of respiratory depression events. Specifically, monitoring equipment per patient may cost less to hospitals than a respiratory depression event, which sometimes leads to rapid response team activation and ICU transfer $[6,16]$. The cost burden of surgical patients with ORADEs are reported to be $\$ 4350-8225[10,21,27,28]$, representing a $27-47 \%$ increase in admission cost. This is consistent with the cost burden of respiratory depression observed in the PRODIGY trial, which were inputs in this model [6].

We also evaluated the effect of patient outliers on modeling results. Model outcomes were consistent across both analyses, with a low break-even point for implementation of continuous respiratory monitoring and decreased annual cost and length of stay following reduction in respiratory depression.

This model has important implications for US hospital administrators, value and analysis committees, and clinical practice stakeholders

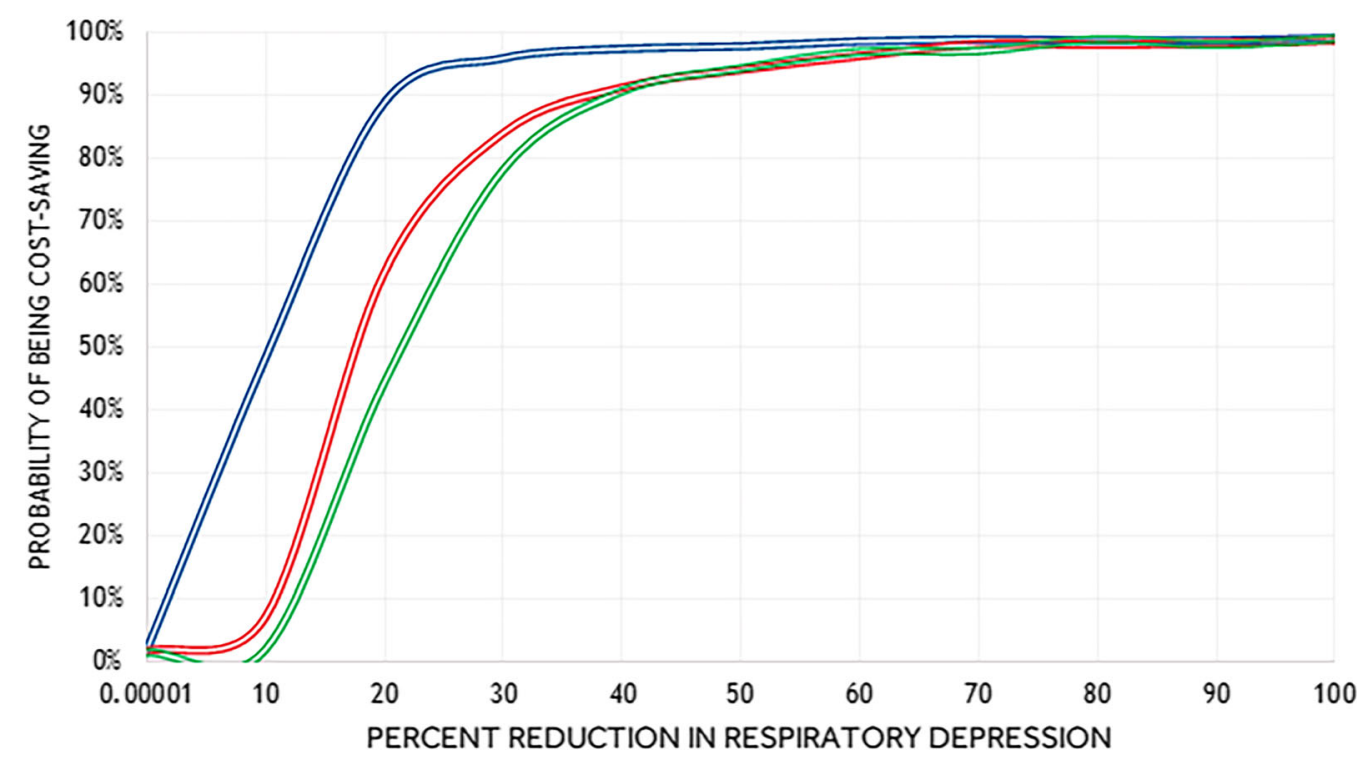

Wigh Risk $=$ High and Intermediate Risk $\quad$ High, Intermediate, and Low Risk

Fig. 3 Probability of cost savings following implementation of continuous pulse oximetry and capnography monitoring on patients with high (blue line), high or intermediate (red line), or high, intermediate, or low (green line) risk for respiratory depression. Model was derived on the basis of the US PRODIGY cohort with cost data available, including outliers 
who are considering (1) whether to continuously monitor hospital general care floor patients receiving opioids, as well as (2) if the monitoring equipment is worth the investment for a subset of this patient population. A critical decision-making element for hospital administrators is identifying appropriate patients to monitor, to balance therapeutic benefit while limiting expenditure. Importantly, sensitivity analysis indicated that across the modeled scenarios, there is a high probability of continuous monitoring being cost saving, with the highest likelihood when monitoring high-risk patients. This analysis may be of particular value to cashstrapped hospitals needing to minimize equipment expenditure while maximizing patient safety.

The clinical utility of implementing continuous respiratory monitoring using pulse oximetry and capnography is supported by multiple studies. In one study, all postoperative patients who had respiratory depression requiring intervention were recognized by capnography, not by pulse oximetry [29]. Additionally, a systematic review and metaanalysis reported that compared to intermittent spot-check monitoring, continuous pulse oximetry decreased the risk of ICU transfer 34\%, and $\mathrm{SpO}_{2}<90 \%>1 \mathrm{~h}$ was 15 times more likely to be detected [24]. With continuous capnography monitoring, $8.6 \%$ more respiratory depression events were detected compared to pulse oximetry monitoring alone, and the odds of respiratory depression detection were 5.83 times higher using capnography vs pulse oximetry [24].

The top strengths of this analysis include its real-world utility to hospital budget holders, its novelty in the literature, and its use of clinical trial data as the basis for the economic model. The hospital budget holder perspective, along with modeling a range of scenarios to minimize budget impact while maximizing patient safety, is pertinent since hospitals are likely to be in financial distress due to the Covid-19 pandemic. This work also adds novelty to the literature, in which we are not aware of any comparable models for respiratory depression. Finally, this model was created on the basis of PRODIGY trial data, which was the largest prospective observational trial of continuous multiparameter monitoring, where waveform data was collected in a blinded manner and subsequently evaluated by an independent adjudication committee.

Our work has some limitations. Our assumption of the number of patients per hospital does not apply to all hospitals. However, we used the Premier ${ }^{\circledR}$ database, which is one of the largest hospital-based discharge databases in the country, to determine the modeled cohort size and represent a median-sized US hospital. While this approach does represent all hospitals, a PRODIGY cost savings calculator to tailor this model to individual hospitals may be helpful but was beyond the scope of this analysis. Second, a $20 \%$ reduction in respiratory depression in the base case is supported by limited literature demonstrating reduced adverse events following implementation of continuous respiratory monitoring. Importantly, our sensitivity analysis eliminated this assumption and evaluated the likelihood of cost savings when all possible scenarios of respiratory depression reduction were modeled from $0 \%$ to $100 \%$. Third, we assume that bedside providers will respond to continuous monitoring alerts, resulting in decreased incidence of respiratory depression, though data on response to continuous monitoring alerts is limited. This model did not include the cost of early intervention to prevent respiratory depression. Finally, the model is based on US PRODIGY patients only, which may limit the applicability of this model internationally, where opioid administration, standard of care practices, and hospital costs vary significantly [30-33].

\section{CONCLUSION}

Although intermittent monitoring has been standard of care for decades, continuous pulse oximetry and capnography monitoring detect more deviations of vital signs and has the potential to increase patient safety on the general care floor $[1,2,24]$. We assessed the investment cost-benefit ratio, and found that compared to intermittent pulse oximetry monitoring, continuous pulse oximetry and 
capnography monitoring of general care floor patients receiving opioids may be a cost-effective and worthwhile investment from the US hospital budget holder perspective. Our results suggest that limiting continuous monitoring to high-risk patients may be the least impactful to budgets, though expansion to intermediateand low-risk patients may offer good value considering the likely reduction in respiratory depression and associated costs.

\section{ACKNOWLEDGEMENTS}

We thank the participants of the PRediction of Opioid-induced respiratory Depression In patients monitored by capnoGraphY (PRODIGY) trial for their valuable contributions.

Funding. Medtronic (Boulder, CO) sponsored the PRODIGY trial and this post hoc analysis, and funded Rapid Service Fees for publication in Advances in Therapy. The authors had final responsibility for the decision to submit for publication. The authors were not paid to write this article by the sponsor or any other agency.

Medical Writing and/or Editorial Assistance. Biostatistical analysis was supported by Paola Di Stefano, MS (Medtronic, Rome, Italy). Rhodri Saunders, PhD of Coreva Scientific (Königswinter, North Rhine-Westphalia Germany) contributed to economic modeling. Medical writing support was provided by Katherine E. Liu, PhD (Medtronic, Minneapolis, $\mathrm{MN}, \mathrm{US})$ and supported by Medtronic.

Authorship. All named authors meet the International Committee of Medical Journal Editors (ICMJE) criteria for authorship for this article, take responsibility for the integrity of the work as a whole, and have given their approval for this version to be published.

Authorship Contributions. Concept and design of work: Ashish K. Khanna, Wolfgang Buhre, Fabio Di Piazza, Leif Saager. Acquisition, analysis, or interpretation of data for the work:
Ashish K. Khanna, Carla R. Jungquist, Wolfgang Buhre, Roy Soto, Fabio Di Piazza, Leif Saager. Drafting of the manuscript: Ashish K. Khanna, Fabio Di Piazza. Critical revision of the manuscript for important intellectual content: Ashish K. Khanna, Carla R. Jungquist, Wolfgang Buhre, Roy Soto, Fabio Di Piazza, Leif Saager. Approval of the final version: Ashish K. Khanna, Carla R. Jungquist, Wolfgang Buhre, Roy Soto, Fabio Di Piazza, Leif Saager.

List of Investigators. PRODIGY investigators included: Ashish K. Khanna, MD; Sergio D. Bergese, MD; Carla R. Jungquist, NP, PhD; Hiroshi Morimatsu, MD, PhD; Shoichi Uezono, MD; Simon Lee, MD; Lian Kah Ti, MBBS, MMed; Richard D. Urman, MD; Robert McIntyre Jr, MD; Carlos Tornero, MD, PhD; Albert Dahan, MD, PhD; Leif Saager, Dr.Med; Toby N. Weingarten, MD; Maria Wittmann, MD; Dennis Auckley, MD; Luca Brazzi, MD, PhD; Morgan Le Guen, MD, PhD; Roy Soto, MD; Frank Schramm, MD; Wolfgang Buhre, MD; and Frank J. Overdyk, MD.

Prior Presentation. A portion of this work was presented as an abstract at the Society for Technology in Anesthesia 2021 Virtual Annual Meeting.

Disclosures. Ashish K. Khanna, Carla R. Jungquist, Wolfgang Buhre, Roy Soto, and Leif Saager (or their institutions) received research support from Medtronic to conduct the PRODIGY trial. In addition, Ashish K. Khanna reports consulting fees from Medtronic, Edwards Lifesciences, and Philips North America and is funded with a Clinical and Translational Science Institute (CTSI) NIH/NCTAS KL2 TR001421 award for a trial on continuous postoperative hemodynamic and saturation monitoring; Carla R. Jungquist reports participation in the Medtronic Nurse Advisory Group; Wolfgang Buhre grants from the European Union and Interreg Consortium, and personal fees from European Society of Anaesthesiology studies (PHOENICS and TETHYS) supported by B Braun Medical and Fresenius Medical Care, and from Medtronic; Fabio Di Piazza reports employment with Medtronic; and Leif Saager 
reports a grant from Merck \& Co. Inc. and consultant fees from Merck \& Co. Inc, The 37 Company, and Ferrer International. Ashish K. Khanna is currently affiliated with the Department of Anesthesiology, Section on Critical Care Medicine, Wake Forest School of Medicine (Winston-Salem, North Carolina, US), and Leif Saager is currently affiliated with the Department of Anesthesiology, University Medical Center Goettingen (Goettingen, Germany).

Compliance with Ethics Guidelines. The PRODIGY trial was conducted following institutional review board or ethics committee approval for each trial site, and in accordance with the Helsinki Declaration of 1964 and its later amendments. The names of all review boards and ethics committees are provided in Table S4 of the Supplementary Material. Written informed consent was collected from all patients before participation in the trial.

Data Availability. All PRODIGY data generated or analyzed during this study are included in this published article/as supplementary information files. The data analyzed from the Premier ${ }^{\circledR}$ Healthcare Database were used under license for the current study and are not publicly available, but data may be available on reasonable request and with the permission of Premier®.

Open Access. This article is licensed under a Creative Commons Attribution-NonCommercial 4.0 International License, which permits any non-commercial use, sharing, adaptation, distribution and reproduction in any medium or format, as long as you give appropriate credit to the original author(s) and the source, provide a link to the Creative Commons licence, and indicate if changes were made. The images or other third party material in this article are included in the article's Creative Commons licence, unless indicated otherwise in a credit line to the material. If material is not included in the article's Creative Commons licence and your intended use is not permitted by statutory regulation or exceeds the permitted use, you will need to obtain permission directly from the copyright holder. To view a copy of this licence, visit http://creativecommons.org/licenses/by$\mathrm{nc} / 4.0 /$.

\section{REFERENCES}

1. Sun Z, Sessler DI, Dalton JE, et al. Postoperative hypoxemia is common and persistent: a prospective blinded observational study. Anesth Analg. 2015;121(3):709-15.

2. Khanna AK, Bergese SD, Jungquist CR, et al. Prediction of opioid-induced respiratory depression on inpatient wards using continuous capnography and oximetry: an international prospective, observational trial. Anesth Analg. 2020;131(4):1012-24.

3. Gupta K, Prasad A, Nagappa M, Wong J, Abrahamyan L, Chung FF. Risk factors for opioid-induced respiratory depression and failure to rescue: a review. Curr Opin Anaesthesiol. 2018;31(1):110-9.

4. Turan A, Chang C, Cohen B, et al. Incidence, severity, and detection of blood pressure perturbations after abdominal surgery: a prospective blinded observational study. Anesthesiology. 2019;130(4): 550-9.

5. Overdyk FJ, Carter R, Maddox RR, Callura J, Herrin $\mathrm{AE}$, Henriquez C. Continuous oximetry/capnometry monitoring reveals frequent desaturation and bradypnea during patient-controlled analgesia. Anesth Analg. 2007;105(2):412-8.

6. Khanna AK, Saager L, Bergese SD, et al. Opioid-induced respiratory depression increases hospital costs and length of stay in patients recovering on the general care floor. BMC Anesthesiol. 2021;21(88):1.

7. Lee LA, Caplan RA, Stephens LS, et al. Postoperative opioid-induced respiratory depression: a closed claims analysis. Anesthesiology. 2015;122(3):659-65.

8. Oderda GM, Evans RS, Lloyd J, et al. Cost of opioidrelated adverse drug events in surgical patients. J Pain Symptom Manage. 2003;25(3):276-83.

9. Kane-Gill SL, Rubin EC, Smithburger PL, Buckley MS, Dasta JF. The cost of opioid-related adverse drug events. J Pain Palliat Care Pharmacother. 2014;28(3):282-93.

10. Urman RD, Seger DL, Fiskio JM, et al. The burden of opioid-related adverse drug events on hospitalized previously opioid-free surgical patients. J Patient Saf. 2021;17(2):e76-83.

11. Taenzer AH, Pyke JB, McGrath SP, Blike GT. Impact of pulse oximetry surveillance on rescue events and 
intensive care unit transfers: a before-and-after concurrence study. Anesthesiology. 2010;112(2): 282-7.

12. Fischer JP, Shang EK, Butler CE, et al. Validated model for predicting postoperative respiratory failure: analysis of 1706 abdominal wall reconstructions. Plast Reconstr Surg. 2013;132(5):826e-e835.

13. Cozowicz C, Olson A, Poeran J, et al. Opioid prescription levels and postoperative outcomes in orthopedic surgery. Pain. 2017;158(12):2422-30.

14. Dunwoody D, Jungquist CR. Monitoring for opioidinduced advancing sedation and respiratory depression: ASPMN membership survey of current practice 2019. 2019 ASPMN Annual Conference, Portland, Oregon. 2019.

15. Jungquist CR, Quinlan-Colwell A, Vallerand A, et al. American Society for Pain Management nursing guidelines on monitoring for opioid-induced advancing sedation and respiratory depression: revisions. Pain Manag Nurs. 2020;21(1):7-25.

16. Stites M, Surprise J, McNiel J, Northrop D, De Ruyter $\mathrm{M}$. Continuous capnography reduces the incidence of opioid-induced respiratory rescue by hospital rapid resuscitation team. J Patient Saf. 2017. https:// doi.org/10.1097/PTS.0000000000000408.

17. Husereau D, Drummond M, Petrou S, et al. Consolidated health economic evaluation reporting standards (CHEERS)-explanation and elaboration: a report of the ISPOR health economic evaluation publication guidelines good reporting practices task force. Value Health. 2013;16(2):231-50.

18. Khanna AK, Overdyk FJ, Greening C, Di Stefano P, Buhre WF. Respiratory depression in low acuity hospital settings-seeking answers from the PRODIGY trial. J Crit Care. 2018;47:80-7.

19. Overdyk FJ, Dowling O, Marino J, et al. Association of opioids and sedatives with increased risk of inhospital cardiopulmonary arrest from an administrative database. PLoS One. 2016;11(2):e0150214.

20. Herzig SJ, Rothberg MB, Cheung M, Ngo LH, Marcantonio ER. Opioid utilization and opioid-related adverse events in nonsurgical patients in US hospitals. J Hosp Med. 2014;9(2):73-81.

21. Shafi S, Collinsworth AW, Copeland LA, et al. Association of opioid-related adverse drug events with clinical and cost outcomes among surgical patients in a large integrated health care delivery system. JAMA Surg. 2018;153(8):757-63.

22. Donohue JM, Kennedy JN, Seymour CW, et al. Patterns of opioid administration among opioidnaive inpatients and associations with postdischarge opioid use: a cohort study. Ann Intern Med. 2019;171(2):81-90.

23. Niroula A, Garvia V, Rives-Sanchez M, et al. Opiate use and escalation of care in hospitalized adults with acute heart failure and sleep-disordered breathing (OpiatesHF study). Ann Am Thorac Soc. 2019;16(9):1165-70.

24. Lam T, Nagappa M, Wong J, Singh M, Wong D, Chung F. Continuous pulse oximetry and capnography monitoring for postoperative respiratory depression and adverse events: a systematic review and meta-analysis. Anesth Analg. 2017;125(6): 2019-29.

25. Steele T, Eidem L, Bond J. Impact of adoption of smart pump system with continuous capnography monitoring on opioid-related adverse event rates: experience from a tertiary care hospital. J Patient Saf. 2019;16:e194.

26. Briggs A, Sculpher M, Claxton K. Decision modelling for health economic evaluation. Oxford: OUP; 2006.

27. Oderda GM, Gan TJ, Johnson BH, Robinson SB. Effect of opioid-related adverse events on outcomes in selected surgical patients. J Pain Palliat Care Pharmacother. 2013;27(1):62-70.

28. Kessler ER, Shah M, Gurschkus SK, Raju A. Cost and quality implications of opioid-based postsurgical pain control using administrative claims data from a large health system: opioid-related adverse events and their impact on clinical and economic outcomes. Pharmacotherapy. 2013;33(4):383-91.

29. McCarter T, Shaik Z, Scarfo K, Thompson LJ. Capnography monitoring enhances safety of postoperative patient-controlled analgesia. Am Health Drug Benefits. 2008;1(5):28-35.

30. Berterame S, Erthal J, Thomas J, et al. Use of and barriers to access to opioid analgesics: a worldwide, regional, and national study. Lancet. 2016;387(10028):1644-56.

31. Burden M, Keniston A, Wallace MA, et al. Opioid utilization and perception of pain control in hospitalized patients: a cross-sectional study of 11 sites in 8 countries. J Hosp Med. 2019;14(12):737-45.

32. Ladha KS, Neuman MD, Broms G, et al. Opioid prescribing after surgery in the United States, Canada, and Sweden. JAMA Netw Open. 2019;2(9): e1910734.

33. Papanicolas I, Woskie LR, Jha AK. Health care spending in the united states and other high-income countries. JAMA. 2018;319(10):1024-39. 